\title{
Clinical utility of Basophil and B-cell biomarkers for monitoring disease activity in food allergy and food oral immunotherapy
}

Theodore Kim ${ }^{1}$, Richard L. Wasserman ${ }^{2}$, Oral Alpan ${ }^{3}$, Atul $\mathrm{Shah}^{4}$ and Douglas Jones ${ }^{5}$

\author{
${ }^{1}$ Allergy Partners of Northern Virginia, Sterling, VA \\ ${ }^{2}$ Allergy Partners of North Texas Research, Dallas, TX \\ ${ }^{3}$ Amerimmune, Fairfax, VA, USA \\ ${ }^{4}$ New York Food Allergy and Wellness Center, Centereach, NY \\ ${ }^{5}$ Tanner Clinic, Layton, UT
}

Corresponding Author:

Theodore Kim, M.D.

Allergy Partners of Northern Virginia

14520 Avion Pkwy, Suite 150

Chantilly, VA 22151

tkim@allergypartners.com 
Once peanut allergy develops, the standard of care has been complete avoidance to prevent potentially fatal systemic allergic reactions.. Over the past decade, reports of case series and placebo-controlled studies have shown that the systematic introduction of small amounts of peanut allergen, followed by gradual increases in dose, could prevent or attenuate systemic reactions ${ }^{1}$. A major concern regarding peanut OIT, that the ability to eat the desensitizing allergen will be temporary and lost if regular consumption ceases has not been resolved and remains an important un-answered question in clinical management of these patients. Although the changes in serum peanut specific IgE and IgG4 shows some correlation with OIT outcomes, it is more likely that both serologic and cellular biomarkers will be needed to accurately predict sustained unresponsiveness and/or immune tolerance ${ }^{2}$. Furthermore, the technical validation and performance of such assays in a clinical laboratory is necessary for the assays to be able to guide clinical care. In line of this thought process, we evaluated two candidate assays; a very well established assay that assesses desensitization and a unique B cell assay that may be a marker for change in atopic status over time.

Basophil activation results in the translocation of lysosomal-associated membrane proteins, including CD63, from a predominantly intracellular location to the cell surface. Therefore, CD63 expression measures cells that have undergone degranulation ${ }^{3}$. The ectonucleotide pyrophosphatase/phosphodiesterase (ENPP)-3, CD203c, is upregulated during activation as well. Activation of circulating basophils has been shown to correlate with clinical disease in several contexts including urticaria, anaphylaxis, asthma, food allergy, autoimmune disease and helminth infection ${ }^{4}$.

We studied 34 patients between 2 and 16 years of age receiving peanut oral immunotherapy (peanut-OIT) in this retrospective data analysis. Collection and analysis of data was approved by Western Institutional Review Board (Protocol: 1-824462-1). Peanut allergy was diagnosed by a clinical history of hives, respiratory and/or gastrointestinal symptoms on ingestion of peanut within the 6 months prior to initiating peanut-OIT, a positive skin test and/or peanut specific $\operatorname{IgE}$ level measurement ( $>5 \mathrm{kU} / \mathrm{L})$ as well as a basophil activation test to peanut. Of the patients with peanut allergy $35 \%$ also had other nut allergies, but no allergies to other foods. Allergic rhinitis and asthma was diagnosed in $90 \%$ and $38 \%$ respectively. Patients were on different stages of 
OIT ranging between $2 \mathrm{mg}$ dose to maintenance therapy. Male to female ratio was 1:1.2. We also collected data from 19 patients with peanut allergy who are not receiving OIT (ages 2-15), 5 healthy controls (ages 4-16) and 5 subjects (ages 7-18) with near equal M:F ratio, who have outgrown peanut allergy as documented by a low peanut specific IgE titer $(<2 \mathrm{kU} / \mathrm{L})$ and passing an oral food challenge. We also included 9 peanut allergic patients with non-releaser basophils (ages 5-14). The non-releaser phenotype can be seen in 15-20\% in the general population as well as in food allergic patients and is characterized by the inability to upregulate CD63 on stimulation with anti-IgE as a positive control.

Basophil surface expression of CD63 and CD203c was examined in response to graded concentrations of peanut allergen (Greer Laboratories). The assays were validated in Amerimmune, a CLIA/CAP approved laboratory. Basophils were identified in peripheral blood as $\mathrm{CD}_{193}{ }^{+} \mathrm{CD}_{123}{ }^{+} \mathrm{IgE}^{+}$cells. Activated basophils were measured by gating on CD63 positive cells as well as the fold change in CD203c mean fluorescence intensity (MFI) compared to the negative control. Whole blood was stimulated with peanut allergen in concentrations ranging between 10-10,000 $\mathrm{ng} / \mathrm{ml}$. Figure 1A shows that the ratio of CD63 to CD203C in peanut-OIT patients is significantly decreased compared to that seen in peanut allergic patients $(\mathrm{p}<0.001)$. The data is from basophils activated with $100 \mathrm{ng} / \mathrm{ml}$ concentration of peanut, which showed the best results, although the patterns for the different concentrations were similar. Although there was a decrease in basophil surface CD63 expression in patients undergoing OIT, CD203c expression showed a decrease, no change or slight increase that did not correlate with the stage of peanut OIT, hence the reason we chose to look at ratios rather than individual surface markers. The stage of the peanut-OIT did not correlate with a decrease in the CD63/203c ratio. It is possible that there is a transition phase for the decrease in $\mathrm{CD} 63 / \mathrm{CD} 203 \mathrm{c}$ ratio, and a prospective study may provide more evidence for the timing of this finding.

We then asked if the changes seen in basophils are accompanied by phenotypic changes in B cells, as one of the goals of OIT is to induce long lasting immunological tolerance. In vitroactivated B cells can downregulate CD73 expression, and inhibit T-cell proliferation and cytokine production $^{5,6}$. Peanut allergic patients showed a significant increase in the expression of CD73, compared to healthy controls or those that have outgrown the peanut allergy, $\mathrm{p}<0.001$ 
(Figure 1B). Patients undergoing peanut-OIT also showed a lower CD73 expression, compared to peanut allergic individuals ( $\mathrm{p}<0.01)$. In healthy controls, B cell CD73 expression shows an age dependent decrease, and the pattern we are seeing here could be the acceleration of the natural outgrowing process induced by peanut OIT (data not shown). The change in CD73 expression did not correlate with the level of suppression in the CD63/CD203 ratio which suggests that changes in B cells are independent of the basophil desensitization (data not shown).

Immune monitoring during immunotherapy of any kind is essential to better understand success, especially in cases where outcomes are not very obvious during the therapy or the therapy itself leads to complications ${ }^{7}$. We now see this in oncology with checkpoint inhibitors where the utility of this treatment modality is creating a new field in medicine that deals with the complications. At the present time, the status of the peanut allergy is determined by oral food challenges with the attendant risk of anaphylaxis. Clinically available and valuable biomarkers need to be part of management in peanut-OIT. 
References:

1- Wasserman RL, Hague AR, Pence DM, et al. Real-World Experience with Peanut Oral Immunotherapy: Lessons Learned From 270 Patients. J Allergy Clin Immunol Pract. 2019; 7:418-426.

2- Chinthrajah RS, Purington N, Andorf S, et al. Sustained outcomes in oral immunotherapy for peanut allergy (POISED study): a large, randomised, double-blind, placebocontrolled, phase 2 study. Lancet. 2019 ;394:1437-1449.

3- Sainte-Laudy J, Sabbah A, Vallon C, Guerin JC. Analysis of anti-IgE and allergen induced human basophil activation by flow cytometry. Comparison with histamine release. Inflamm Res 1998; 47:401-8.

4- Hoffmann HJ, Knol EF, Ferrer M, et al. Pros and Cons of Clinical Basophil Testing (BAT). Curr Allergy Asthma Rep 2016; 16:56.

5- Saze Z, Schuler PJ, Hong CS, Cheng D, Jackson EK, Whiteside TL. Adenosine production by human B cells and B cell-mediated suppression of activated T cells. Blood. 2013;122: 9-18.

6- Alpan O, Rudomen G, Matzinger P. The role of dendritic cells, B cells, and M cells in gut-oriented immune responses. J Immunol. 2001; 166:4843-52.

7- Bakacs T, Moss RW, Kleef R, Szasz MA, Anderson CC.

Exploiting autoimmunity unleashed by low-dose immune checkpoint blockade to treat advanced cancer. Scand J Immunol. 2019 Oct 7:e12821. doi: 10.1111/sji.12821. 


\section{Figure 1}

A

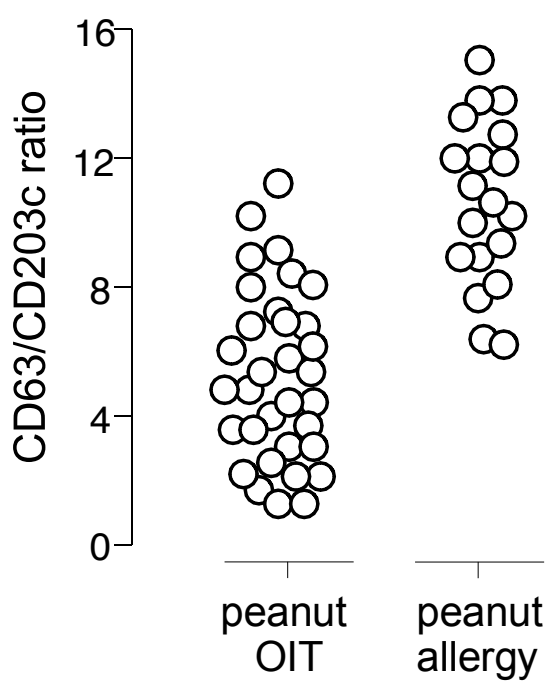

B

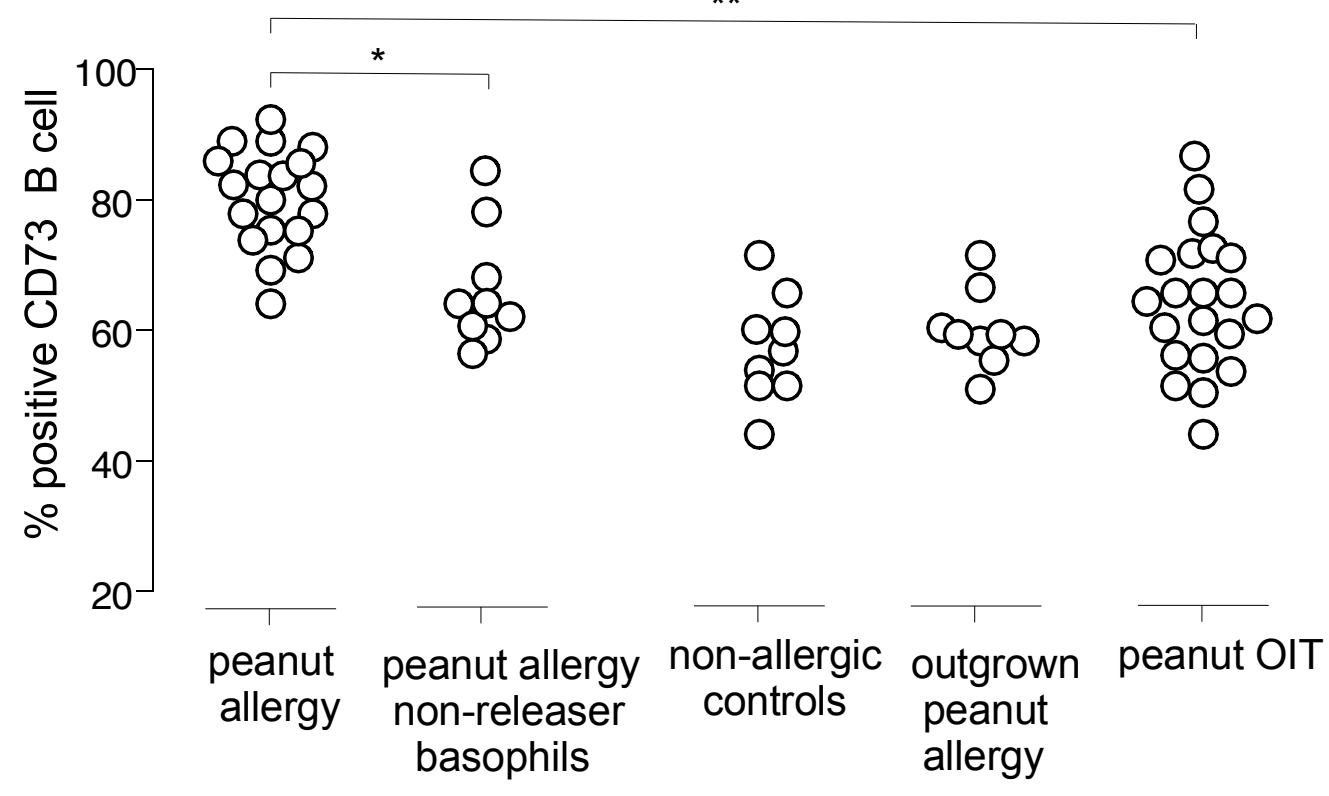

Figure 1. a) CD63/CD203c ratio. CD203c is reported as fold change in mean fluorescence intensity on basophils. CD63 is reported as percent positive basophils that stain positive for CD63. b) CD73 expression on B cells. 